\title{
Spiking Behavior in a Noise-Driven System Combining Oscillatory and Excitatory Properties
}

\author{
V. A. Makarov, ${ }^{1}$ V. I. Nekorkin, ${ }^{1,2}$ and M. G. Velarde ${ }^{1}$ \\ ${ }^{1}$ Instituto Pluridisciplinar, Universidad Complutense, Paseo Juan XXIII, 1, Madrid, 28040, Spain \\ ${ }^{2}$ Radiophysical Department, Nizhny Novgorod St. University, Gagarin Avenue, 23, Nizhny Novgorod, 603600, Russia
}

(Received 29 September 2000)

\begin{abstract}
We show that firing activity (spiking) can be regularized by noise in a FitzHugh-Nagumo (FHN) neuron model when operating slightly beyond the supercritical Hopf bifurcation (in the "canard" region). We also provide the conditions for imperfect phase locking between interspike intervals and low amplitude quasiharmonic oscillations. For the imperfect phase locking no need exists of an external signal as it follows from the FHN intrinsic dynamics.
\end{abstract}

DOI: 10.1103/PhysRevLett.86.3431

Recent experimental data on single neurons and neuron networks [1-5] have alerted us to the significant role played by noise in the evolution of excitable systems, in general. It has been shown in particular that neurons may use noise to their advantage by enhancing their sensitivity near a preferred phase to detect external stimuli. On the other hand, although action potentials and interspiking time intervals are used by neurons for transmitting coded information [6], yet spiking series come with a nonnegligible amount of noise.

From the theoretical side, particular attention has been given to models like FitzHugh-Nagumo equations and other simplified albeit significant approximations to reality [6-17]. It has been shown that the output of a nonlinear system can be optimized or tuned to a given purpose by a suitable noise signal. Furthermore, it is known in various fields of science and engineering the significant role played by stochastic resonance [18-20], aperiodic stochastic resonance [10], and coherent resonance [14]. For instance, a significant improvement in human sensory perception mediated by stochastic resonance has been observed [4]. Another example is experiments on single neurons in the visual cortex of a cat [5] showing that an interspike interval histogram (ISIH) data can form a decaying sequence of peaks located at equidistant distributed positions. Experiments with inferior olive neurons demonstrate a similar dynamical behavior, within statistical significance, in their capability of providing rhythmic activity in the cerebellum [1-3,21].

Recently, Pikovsky and Kurths [14] using the FitzHughNagumo (FHN) model [7] have shown that stochastic resonancelike behavior can be observed even without an external signal. The coherence of oscillations produced by a system is maximum at moderate noise value. Similar results were obtained for the Hodgkin-Huxley equations [15]. Baltanas and Casado [16] using the FHN model driven by quasimonochromatic noise (QMN) have shown an imperfect phase locking between the interspike intervals and the fundamental period of QMN. The resulting ISIH has maxima at integer multiples of the fundamental period. Generally, the FHN model $[7,8]$ is considered in two regimes:
PACS numbers: 84.35.+i, 05.40.Ca, 05.45.-a, 87.19.La

(i) a relaxation type limit cycle is the only attractor with strongly nonlinear oscillations in the system or

(ii) a steady state is the only attractor and relatively small perturbations can give rise to a large excursion (the excitation loop) or a spike, as called in neurodynamics, and then the system returns back to the steady state.

Here we shall consider the case intermediate between (i) and (ii), when the creation of the limit cycle in the FHN model occurs associated to the canard phenomenon $[22,23]$. In the parameter region "between" cases (i) and (ii), slightly beyond the supercritical Hopf bifurcation the FHN model can generate low amplitude quasiharmonic oscillations albeit remaining excitable (Fig. 1). Indeed, sufficiently strong perturbations may activate pulse response (spike) and then the system returns to the low amplitude quasiharmonic oscillations which are thus called "subthreshold" oscillations. In this note we study the responses to white Gaussian noise of the FHN model in this initially "silent" parameter region.

Let us consider the system of differential equations

$$
\begin{gathered}
\varepsilon \frac{d u}{d t}=u(u-a)(1-u)-v, \\
\frac{d v}{d t}=g(u-b)+\sqrt{2 D} \xi(t),
\end{gathered}
$$

where $\varepsilon \ll 1$ is a smallness parameter, and $u$ and $v$ refer to voltage and recovery variables, respectively. The

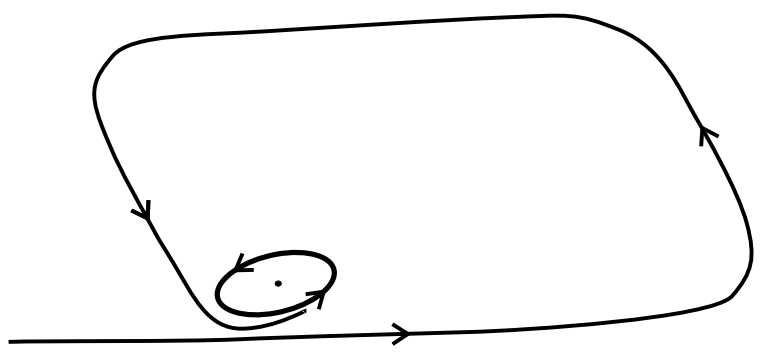

FIG. 1. Sketch illustrating coexistence of oscillatory (stable limit cycle) and excitatory (long path curve) properties in the FHN model slightly beyond the supercritical Hopf bifurcation. 
term $\xi(t)$ represents a Gaussian delta-correlated noise with zero mean. The rhs of Eq. (1b) consists of a monotonically increasing function, $g(x)$, satisfying the conditions: $g(0)=0, g^{\prime}(x)>0$, and $g^{\prime}(0)=1$. For $g(x) \equiv x$ the system (1a),(1b) is the original FHN model [7]. We have modified the standard equations to get flexibility to tune independently the time scales of the oscillation displayed by Eqs. (1a) and (1b) as it will be further emphasized. Here, for numerical analysis we shall use

$$
g(x)=k_{1} x^{2}+k_{2}\left(1-\exp \left[-\frac{x}{k_{2}}\right]\right),
$$

where $k_{1}$ and $k_{2}$ are new parameters chosen such that the condition $g^{\prime}(x)>0$ is satisfied for any $x$.

As already mentioned we shall consider the system (1a),(1b) near the supercritical Hopf bifurcation $b \geqq b^{*}$, with

$$
b^{*}=\frac{1+a-\sqrt{1-a+a^{2}}}{3} .
$$

The deterministic system generates quasiharmonic oscillations (Fig. 2a) whose corresponding limit cycle remains in the thin layer of "slow" motions and hence does not break the excitable property of the FHN model. Thus for such parameter values the FHN model has both oscillatory and excitatory properties. Because of the constraint $\left[g(0)=0, g^{\prime}(0)=1\right]$ the period of subthreshold oscillations near the bifurcation point (3) is $T_{\text {sth }} \approx 2 \pi \sqrt{\varepsilon}$, and depends on $\varepsilon$ only. The function $g(x)$ helps to tune in the system (1a),(1b) the time scales of the spikes independently of the period of subthreshold oscillations. By playing with $k_{1}$ and $k_{2}$ we can change the value of $d v / d t$ in the domains of slow motion and, consequently, the time intervals spent by a trajectory in these domains, whose approximate values are (a)

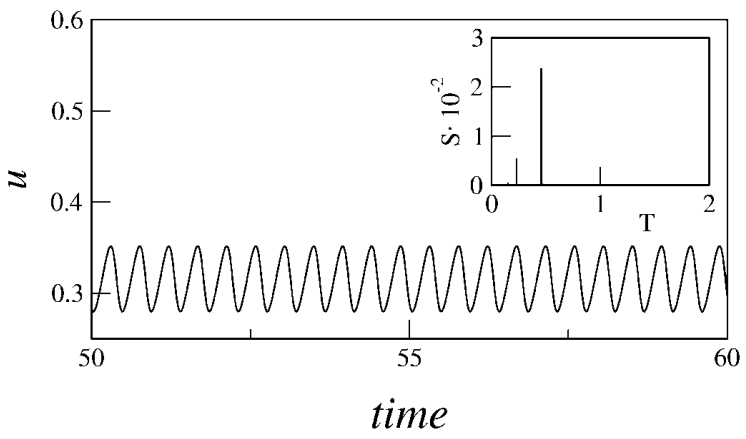

(b)

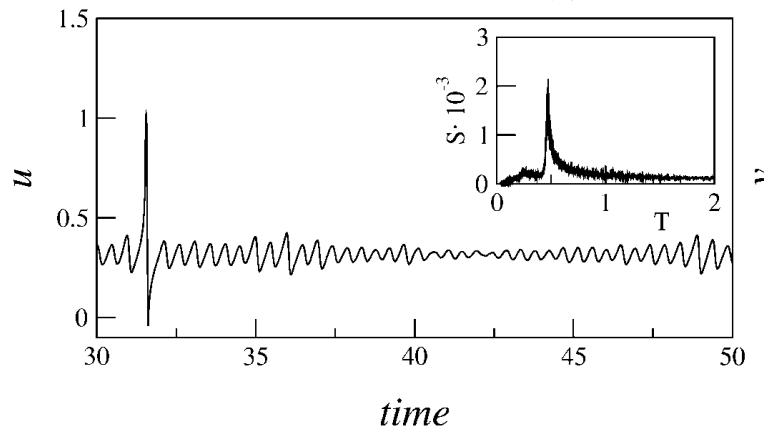

(c)

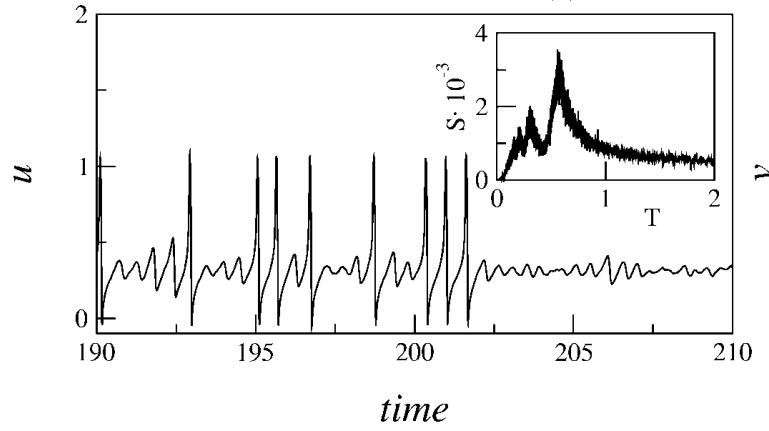

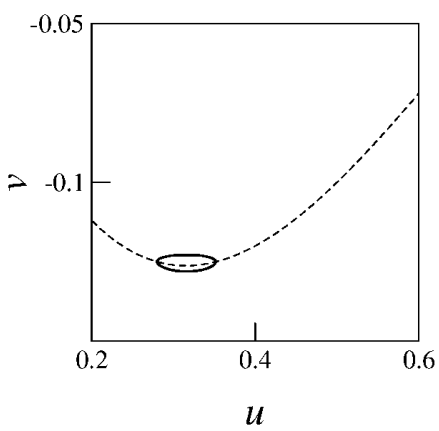
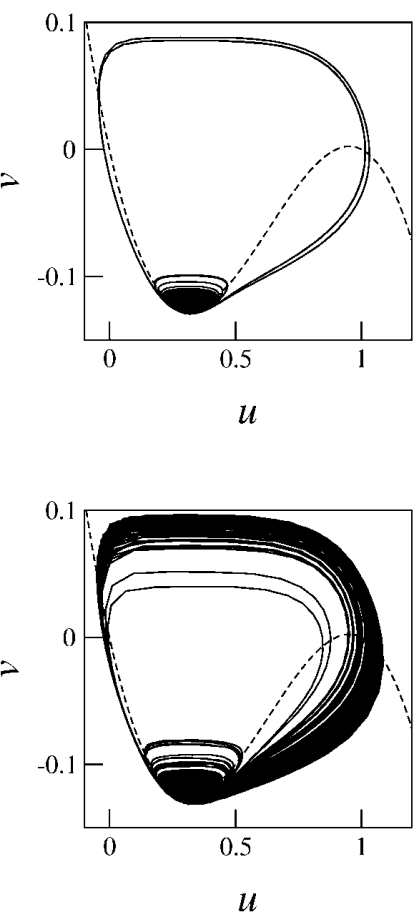

FIG. 2. Time series of the voltagelike variable, $u$, and corresponding power spectra and phase portraits for different values of noise intensity: (a) $D=0$, (b) $D=0.6 \times 10^{-6}$, (c) $D=0.6 \times 10^{-5}$. Dashed curve corresponds to the "slow" motions $v=f(u)$. 


$$
T_{\mathrm{exc}}=\frac{4\left(1-a+a^{2}\right)^{3 / 2}}{27 g\left(1-b^{*}\right)}, \quad T_{\mathrm{ref}}=-\frac{4\left(1-a+a^{2}\right)^{3 / 2}}{27 g\left(-b^{*}\right)} .
$$

Clearly, $k_{1}$ and $k_{2}$ affect both the duration of the excitation interval and the refractory period following a spike. Moreover, $k_{1}$ and $k_{2}$ mostly govern $T_{\text {exc }}$ and $T_{\text {ref }}$, respectively. Thus using appropriate values of $k_{1}$ and $k_{2}$ we can alter the duration of the spikes. The price we pay to have such flexibility is a rather strong deviation of the trajectories from the slow motion curve, $v=f(u)$, for high values of $u$ (Fig. 2).

For illustration, we fix parameter values $\varepsilon=0.005$ and $a=0.9$. With this choice the period of subthreshold oscillations is $T_{\text {sth }} \approx 0.44$ and the critical value of $b^{*}$ is approximately 0.315 . We chose $b=0.316$, i.e., slightly beyond the bifurcation. Thus the low amplitude limit cycle (subthreshold oscillations) is reached from any point of the phase space of the noise-free system. Using $k_{1}=7.0$ and $k_{2}=0.08$ we get that the two time scales in a spike are approximately equal, $T_{\mathrm{exc}} \approx T_{\text {ref }} \approx 0.04$. Thus the duration of a spike, $\left(T_{\mathrm{exc}}+T_{\text {ref }}\right)$, was chosen much shorter than the above indicated period of subthreshold oscillations, $T_{\text {sth }}$. Such relation is typical to an inferior olive neuron.

We have integrated the system (1a),(1b) using Euler's method [24]. The response of the FHN model to various intensity levels of the white Gaussian noise is shown in Fig. 2. In the deterministic case (Fig. 2a) the system generates oscillations with period $T_{f} \approx 0.45$ which is very close to the linear approximation $T_{\mathrm{sth}}=0.44$. For low enough noise intensity (Fig. 2b) the output of the FHN model is oscillatory with the power spectrum peaked around $T_{f}$. The quantity $u$ performs fast oscillations with relatively slow varying amplitude. Spikes are very rare. For a moderate noise intensity level (Fig. 2c) the spectrum widens and has relatively high peaks in the short time interval. This is due to increasing spike rate and, consequently, to a higher contribution of the fast motions. For strong enough noise intensity the output is highly irregular.

First let us check the influence of subthreshold oscillations in the phenomenon of coherence resonance reported in [14]. Averaging results of 30 runs we compute the normalized autocorrelation function, $C(\tau)$, of $v(t)$. Then we calculate the characteristic correlation time

$$
\tau_{c}=\int_{0}^{\infty} C^{2}(\tau) d t
$$

Figure 3 shows this quantity as a function of noise intensity level. The characteristic correlation time has a rather well-pronounced maximum at moderate noise intensity level $\left(D \approx 1.54 \cdot 10^{-5}\right)$. This indicates the existence of coherence resonance in the system in agreement with the results reported in [14]. However, at very low noise

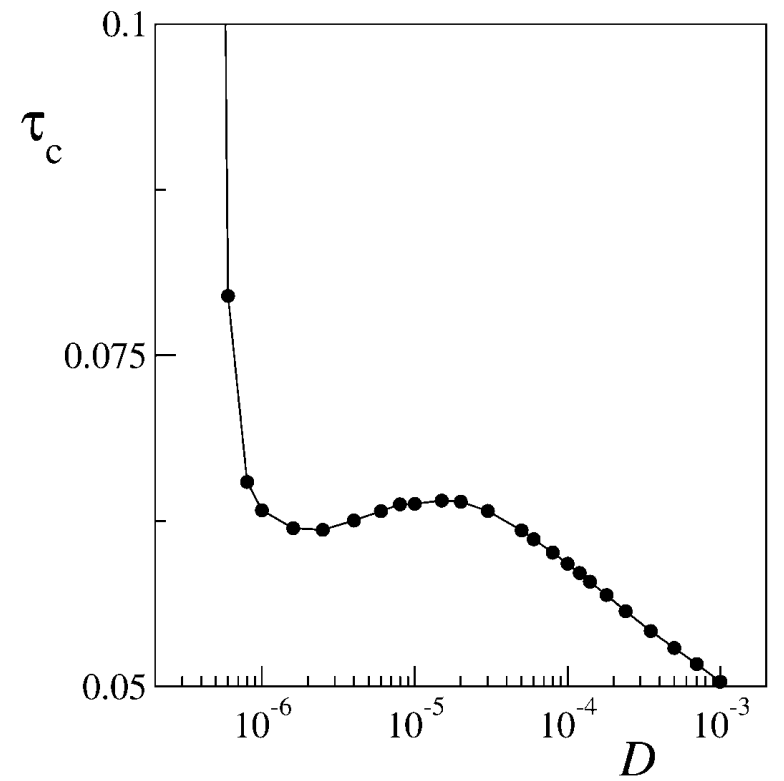

FIG. 3. Characteristic correlation time vs noise intensity computed by using (5).

intensity the value of $\tau_{c}$ sharply increases. The rationale behind this new result goes as follows. For low enough noise intensity level, $D$, spikes appear very rarely (Fig. 2b) and, practically, do not contribute to the correlation function. Hence we can omit them and consider the influence of the noise on the limit cycle oscillator (limited to subthreshold oscillations only). Decreasing $D$ to zero, the correlation function can be approximated by $C(\tau) \propto \exp (-D) \cos \omega \tau$ instead of going to zero as in the parameter region (ii), and hence for $D \rightarrow 0, \tau_{c} \rightarrow \infty$ (Fig. 3).

The stochastic signals shown in Figs. $2 \mathrm{~b}$ and $2 \mathrm{c}$ are similar to the traces reported in [16] when studying the influence of a quasimonochromatic noise on the FHN model in the parameter region (ii). The important feature is that the voltagelike variable fires when the system passes the maxima of subthreshold oscillations and, consequently, has a maximum of probability to escape them. Thus the system has a preferred phase value to fire. To check this ability we calculated ISIH averaging over 200 realizations with approximately 15000 spikes per run. We count a spike event if the voltagelike variable, $u(t)$, crosses from below the threshold value $u_{0}=0.7$. Figure 4 shows ISIH for three values of noise intensity level, $D$. All histograms have clear equidistant peaks. This supports our hypothesis about the existence of a preferable phase for subthreshold oscillations. Increasing the noise intensity level the first peak corresponding to firing at each oscillation period increases while the others decrease and become blurred.

Thus in the parameter region considered above besides the coherence resonance discovered in [14], the FHN model exhibits imperfect phase locking between the interspike intervals and the fundamental period of subthreshold oscillations. A similar phenomenon was reported in [16] 

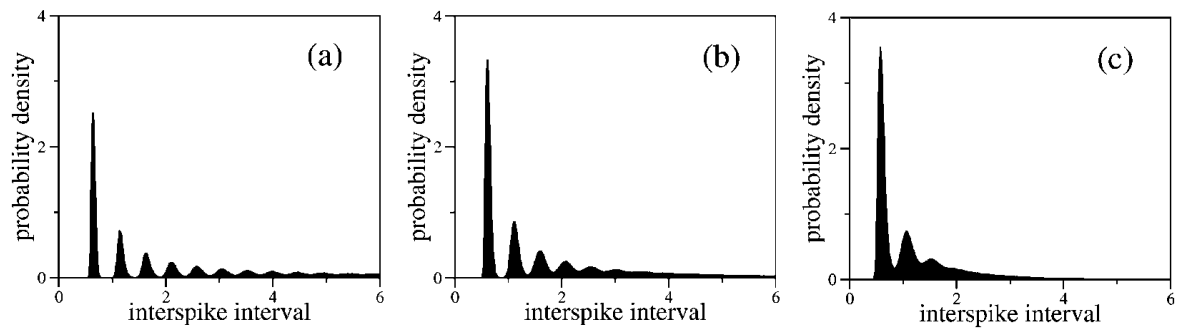

FIG. 4. Interspike interval histograms corresponding to three different noise intensity levels: (a) $D=2 \times 10^{-6}$, (b) $D=6 \times$ $10^{-6}$, (c) $D=2 \times 10^{-5}$.

but here in our case no additional second order stochastic differential equation for generating a $\mathrm{QMN}$ is needed. Instead we have shown that the system can achieve different behaviors due to its intrinsic dynamics by suitably varying the values of its parameters $\left(\epsilon, k_{1}\right.$, and $\left.k_{2}\right)$. The ISIHs shown on Fig. 3 strongly depend on the relation between frequency of subthreshold oscillations and spike width (regulated by $\epsilon, k_{1}$, and $k_{2}$ ). For parameter values different from those considered above the firing pattern is drastically different, thus illustrating the richness of the dynamics of the noise-driven FHN model.

Let us conclude by saying that experimental traces recorded from a slice of inferior olive (see in particular Figs. 6A and 8A in [2] and Fig. 5 in [1]) show behavior reminiscent of the above described for the noise-driven FHN model. In particular, to have subthreshold oscillations suffices to require that the value of $b$ be close enough to the bifurcation value (3). Note that for $b<b^{*}$ the system has a stable focus, hence it is capable of producing damped oscillations that may eventually become sustained by a suitable noise. For models of neuron networks it has been shown that oscillations can appear due to loose coupling between neurons $[25,26]$. This supports the hypothesis that neurons may be, generally, operating at the edge of instability, close to bifurcation points, and small perturbations, for example due to a noisy environment, can give rise to firing activity that in turn, and this is the crucial point, can be regularized by their intrinsic dynamics.

Motivation for this research came from discussions with Dr. R. R. Llinas, Dr. A. Fdez. de Molina, Professor H. Haken, and Professor G. Nicolis. Research supported by the Spanish Ministry of Science and Technology (Grant No. PB96-599), by the Programa Catedra of the BBV Foundation (Spain), and by the Russian Foundation (Grant No. 00-02-16400). V. A. M. benefited from support from the Spanish Ministry of Science and Technology.

[1] R. Llinas and Y. Yarom, J. Physiol. 376, 163 (1986).

[2] L. S. Benardo and R.E. Foster, Brain Res. Bull. 17, 773 (1986).
[3] K. Sasaki, J. M. Bower, and R. Llinas, Eur. J. Neurosci. 1, 572 (1989).

[4] J. J. Collins, T. T. Imhoff, and P. Grigg, Nature (London) 383, 770 (1996).

[5] R. M. Siegel, Physica (Amsterdam) 42D, 385 (1990).

[6] H. Haken, Principles of Brain Functioning (SpringerVerlag, Berlin, 1996).

[7] R. FitzHugh, Biophys. J. 1, 445 (1961).

[8] F. C. Hoppensteadt, An Introduction to the Mathematics of Neurons (Cambridge Univ. Press, NY, 1986).

[9] K. Wiesenfeld, D. Pierson, E. Pantazelou, C. Dames, and F. Moss, Phys. Rev. Lett. 72, 2125 (1994).

[10] J. J. Collins, C. C. Chow, and T. T. Imhoff, Phys. Rev. E 52, R3321 (1995).

[11] J. Milton, Dynamics of Small Neural Populations (American Math. Soc., Providence, Rhode Island, 1996).

[12] A. Longtin, Phys. Rev. E 55, 868 (1997).

[13] C. Eichwald and J. Walleczek, Phys. Rev. E 55, R6315 (1997).

[14] A. S. Pikovsky and J. Kurths, Phys. Rev. Lett. 78, 775 (1997).

[15] S. G. Lee, A. Neiman, and S. Kim, Phys. Rev. E 57, 3292 (1998).

[16] J.P. Baltanas and J.M. Casado, Physica (Amsterdam) 122D, 231 (1998).

[17] C. Hauptmann, F. Kaiser, and C. Eichwald, Int. J. Bifurcation Chaos Appl. Sci. Eng. 6, 1159 (1999).

[18] R. Benzi, A. Sutera, and A. Vulpiani, J. Phys. A 14, L453 (1981).

[19] C. Nicolis and G. Nicolis, Tellus 33, 225 (1981).

[20] L. Gammaitoni, P. Hanggi, D. Jung, and F. Marchesoni, Rev. Mod. Phys. 70, 223 (1998).

[21] R. R. Llinas and K. D. Walton, in The Synaptic Organization of the Brain, edited by G. M. Shepherd (Oxford Univ. Press, Oxford, 1998), Chap. 7.

[22] F. Dumortier, in Bifurcations and Periodic Orbits of Vector Fields, edited by D. Schlomiuk (Kluwer Academic Press, Dordrecht, 1993), pp. 19-73.

[23] P. Glendinning, Stability, Instability and Chaos: An Introduction to the Theory of Nonlinear Differential Equations (Cambridge Univ. Press, NY, 1994), Chap. 8.10.

[24] R. F. Fox, I. R. Gatland, R. Roy, and G. Vemuri, Phys. Rev. A 38, 5938 (1988).

[25] J. M. Kowalski, G. L. Albert, B. K. Rhoades, and G. W. Gross, Neural Netw. 5, 805 (1992).

[26] Y. Manor, J. Rinzel, I. Segev, and Y. Yarom, J. Neurophysiol. 77, 2736 (1997). 